Case Report

\title{
Rhodotorula Endogenous Endophthalmitis: A Novel Harbinger of the Injection Drug Epidemic in the United States
}

\author{
Preston M. Luong, ${ }^{1}$ Basilio Kalpakian, ${ }^{1,2}$ Lawrence J. Jaeger, ${ }^{2}$ Timothy Lahey, ${ }^{1,3}$ \\ Christopher B. Chapman, ${ }^{1,4}$ and Michael E. Zegans ${ }^{1,4}$ \\ ${ }^{1}$ Geisel School of Medicine at Dartmouth, Hanover, NH, USA \\ ${ }^{2}$ Section of Ophthalmology, Dartmouth-Hitchcock Keene, Keene, NH, USA \\ ${ }^{3}$ Section of Infectious Disease, Department of Medicine, Dartmouth-Hitchcock Medical Center, Lebanon, NH, USA \\ ${ }^{4}$ Section of Ophthalmology, Department of Surgery, Dartmouth-Hitchcock Medical Center, Lebanon, NH, USA
}

Correspondence should be addressed to Michael E. Zegans; michael.e.zegans@hitchcock.org

Received 6 March 2017; Accepted 27 March 2017; Published 5 April 2017

Academic Editor: Raul Colodner

\begin{abstract}
Copyright (C) 2017 Preston M. Luong et al. This is an open access article distributed under the Creative Commons Attribution License, which permits unrestricted use, distribution, and reproduction in any medium, provided the original work is properly cited.

Endogenous endophthalmitis is a rare but feared infectious ocular complication of injection drug use (IDU). The recent opioid epidemic in the United States threatens to increase the incidence of this disease. We report the first case of endogenous endophthalmitis in the United States caused by the emerging fungal pathogen Rhodotorula in an injection drug user which led to no light perception vision (NLP). Worldwide experience with Rhodotorula endogenous endophthalmitis is limited, but existing cases suggest infection by this particular fungal genus has a grim prognosis.
\end{abstract}

\section{Introduction}

Mortality from the United States opioid epidemic has tripled to 9 per 100,000 persons since 2000 [1]. The health problems associated with injection drug use (IDU) are legion, with endogenous endophthalmitis among the most debilitating. Endogenous endophthalmitis can result from blood stream dissemination of organisms that contaminate injected drugs and may occur alone or secondary to endocarditis [2].

Fungi and bacteria both cause endogenous endophthalmitis with the most common pathogens being streptococci, staphylococci, and Candida spp. [2]. The rise of IDU raises the opportunity for uncommon microbes to be implicated as causative agents. Rhodotorula is a pinkpigmented yeast found widely in soil, water, and air. It has been isolated in infections from catheters and other foreign bodies [3]. A 2008 systematic review of all Rhodotorula infections in the medical literature cited only two cases of Rhodotorula endogenous endophthalmitis worldwide [3]. To our knowledge, no other case has been reported since. Here we present the first described case of Rhodotorula-associated endogenous endophthalmitis in the United States.

\section{Case Report}

A 21-year-old man with four days of left sided vision loss, eye pain, and conjunctival injection was examined. He reported floaters for two weeks. He was previously healthy but admitted to IDU. He had no recent surgeries, dental work, or intravenous catheters.

Upon examination in the left eye, the best-corrected visual acuity was counting fingers at one foot. Slit lamp examination revealed $4 / 4$ conjunctival injection, $4 / 4$ anterior chamber cells with hypopyon, and posterior synechiae. Ophthalmoscopic exam yielded a hazy view of two white collections in the posterior vitreous. The right eye was normal upon examination. He underwent pars plana vitrectomy with intravitreal injection of amphotericin B $(5 \mathrm{mcg})$, ceftazidime $(2.25 \mathrm{mg})$, and vancomycin $(1.0 \mathrm{mg})$. He was discharged to home with atropine $(1 \%)$, moxifloxacin $(0.5 \%)$, prednisolone $(1 \%)$, and oral azithromycin $(250 \mathrm{mg})$. No microbes were isolated from blood and vitreous cultures nor did Gram stain or calcofluor white reveal any organisms.

At one-month follow-up, although pain had completely subsided, visual acuity remained unchanged at hand motion 
TABLE 1: Rhodotorula endogenous endophthalmitis cases to present.

\begin{tabular}{lccccccc}
\hline Year & Country & Age/sex & Coinfection & History of IDU & Affected eye & Treatment & Visual outcome \\
\hline $2001[4]$ & Italy & $27 / \mathrm{M}$ & Hepatitis C & Positive & Right & IA + oral ketoconazole & Uncertain light perception \\
$2002[5]$ & Canada & $26 / \mathrm{M}$ & HIV & Positive & Right & IA + SA & Enucleation \\
$2011^{*}$ & USA & $21 / \mathrm{M}$ & Hepatitis C & Positive & Left & IA + oral posaconazole & No light perception \\
\hline
\end{tabular}

* The current case presented

HIV: Human Immunodeficiency Virus

IA: intravitreal amphotericin B, SA: systemic amphotericin B.

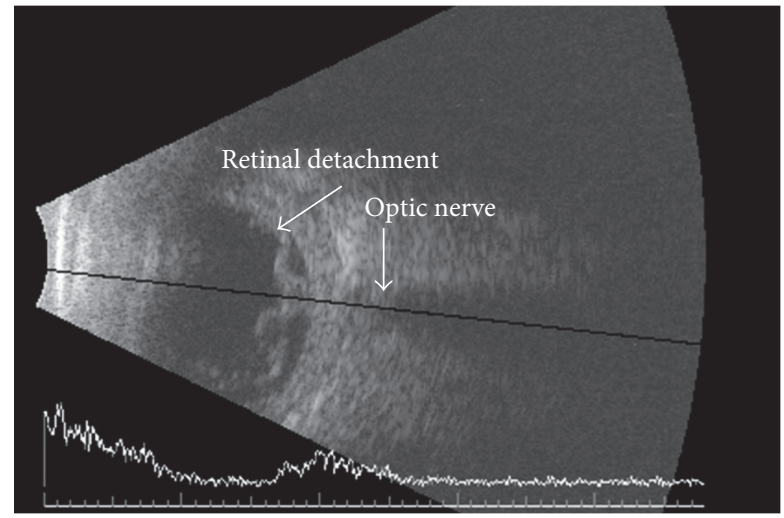

Figure 1: OS B-scan 10 days after final vitrectomy demonstrating funnel retinal detachment tethered to optic nerve head.

along with nonclearing vitritis and a posterior pole white mass suggestive of an abscess. A second pars plana vitrectomy with abscess debridement and intravitreal injections of ceftazidime $(2.25 \mathrm{mg})$ and vancomycin $(1.0 \mathrm{mg})$ was performed. He was again discharged with moxifloxacin $(0.5 \%)$ and prednisolone (1\%) drops. A culture of the abscess identified colonies of the Rhodotorula species susceptible to amphotericin B and posaconazole. The specific species was narrowed to either $R$. mucilaginosa or $R$. glutinis through observation of morphology, pigmentation, and biochemical testing via the BioMerieux Vitek II Yeast Identification Card. Oral posaconazole $(800 \mathrm{mg}$ ) was prescribed for 14 days. Unfortunately, a funnel retinal detachment developed 10 days after surgery (Figure 1). This detachment was deemed inoperable by two retinal specialists because of extensive retinal ischemia. No clinical recurrence of infection occurred however. His most recent follow-up visit three years later revealed no light perception in a left phthisis bulbi.

Table 1 relates the present case, the only report of Rhodotorula endogenous endophthalmitis in the United States, to the two other known cases $[4,5]$.

\section{Discussion}

The visual prognosis is poor in the limited worldwide clinical experience with Rhodotorula endogenous endophthalmitis: all three known cases including ours were associated with IDU and resulted in worse than light perception vision. The most common fungal endogenous endophthalmitis, caused by C. albicans, rarely results in visual loss of this severity [2].
The prognosis of Rhodotorula endogenous endophthalmitis stands in contrast to the better prognoses seen in other eye infections such as scleritis and keratitis caused by this rare yeast $[6,7]$. Although vitreous culture is the gold standard for the diagnosis of fungal endogenous endophthalmitis, its sensitivity varies greatly, with values reported as low as $40 \%$ [8]. Our case underlines the importance of resampling and retreating eyes with suspected endogenous endophthalmitis as microbes may be sequestered and inaccessible in an abscess.

Our patient is the first described case of Rhodotorula endogenous endophthalmitis in the United States. Increasing opioid use in the last decade portends a rise of endogenous endophthalmitis and the emergence of rare infectious agents and poor visual outcomes. Rhodotorula should be considered in the differential diagnosis of IDU patients with sudden profound visual loss, especially if response to initial therapy is suboptimal. Given the association with endocarditis, blood cultures should be drawn in all patients with endogenous endophthalmitis prior to the institution of broad spectrum antibiotics. Hepatitis C and HIV coinfection is also common and too should be evaluated in these cases. The prognosis in Rhodotorula endogenous endophthalmitis is grave, so increased investment in prevention of IDU is critical.

\section{Conflicts of Interest}

The authors declare that there are no conflicts of interest regarding the publication of this paper.

\section{References}

[1] R. A. Rudd, N. Aleshire, J. E. Zibbell, and R. Matthew Gladden, "Increases in drug and opioid overdose deaths-United States, 2000-2014," Morbidity and Mortality Weekly Report, vol. 64, no. 50-51, pp. 1378-1382, 2016.

[2] T. Ness, K. Pelz, and L. L. Hansen, "Endogenous endophthalmitis: microorganisms, disposition and prognosis," Acta Ophthalmologica Scandinavica, vol. 85, no. 8, pp. 852-856, 2007.

[3] F. F. Tuon and S. F. Costa, "Rhodotorula infection. A systematic review of 128 cases from literature," Revista Iberoamericana de Micologia, vol. 25, no. 3, pp. 135-140, 2008.

[4] A. Pinna, F. Carta, S. Zanetti, S. Sanna, and L. A. Sechi, "Endogenous Rhodotorula minuta and Candida albicans endophthalmitis in an injecting drug user," British Journal of Ophthalmology, vol. 85, no. 6, p. 754, 2001.

[5] A. B. Merkur and W. G. Hodge, "Rhodotorula rubra endophthalmitis in an HIV positive patient," British Journal of Ophthalmology, vol. 86, no. 12, pp. 1444-1445, 2002. 
[6] J. Giovannini, R. Lee, S. X. Zhang, A. S. Jun, and K. S. Bower, "Rhodotorula keratitis: a rarely encountered ocular pathogen," Case Reports in Ophthalmology, vol. 5, no. 3, pp. 302-310, 2014.

[7] Z. S. Pradhan and P. Jacob, "Management of Rhodotorula scleritis," Eye, vol. 26, no. 12, p. 1587, 2012.

[8] K. Liu, F. Fang, and H. Li, "Reliability of vitreous histological detection of pathogenic fungi in the diagnosis of fungal endophthalmitis," Eye, vol. 29, no. 3, pp. 424-427, 2015. 


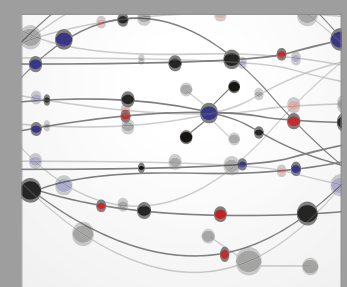

The Scientific World Journal
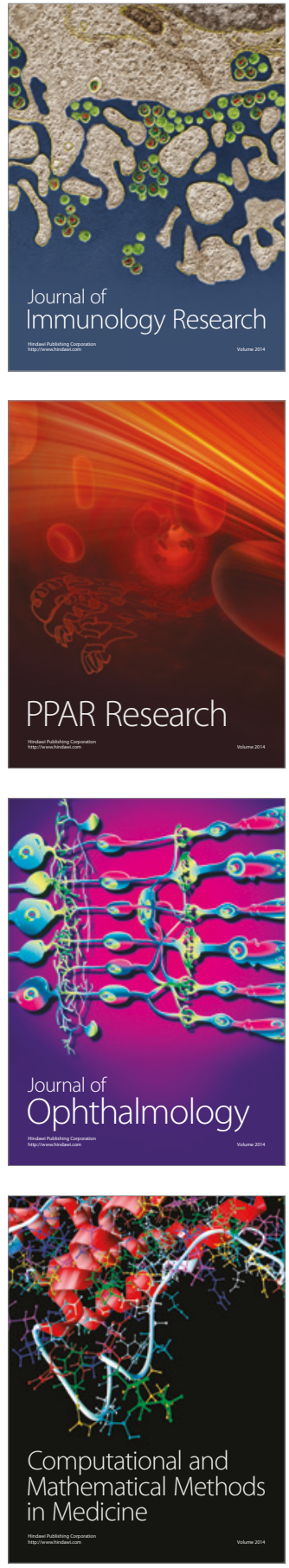

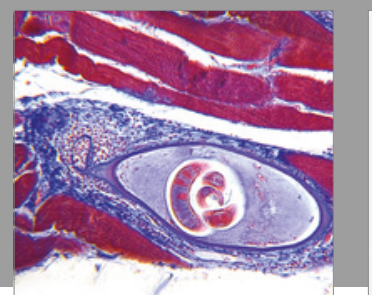

Gastroenterology Research and Practice
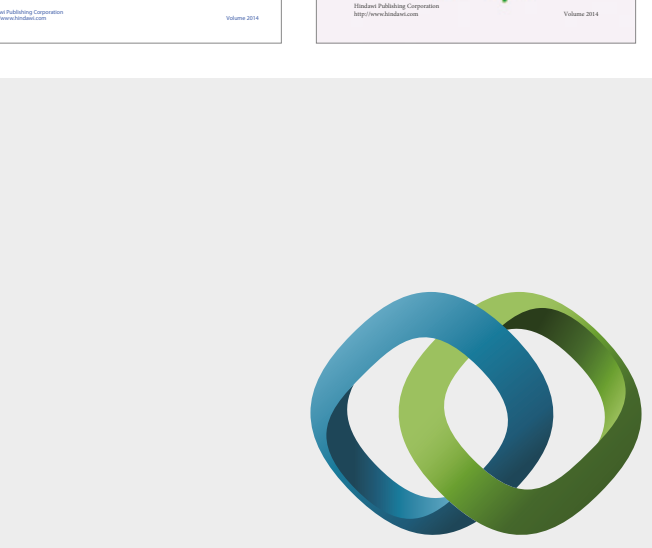

\section{Hindawi}

Submit your manuscripts at

https://www.hindawi.com
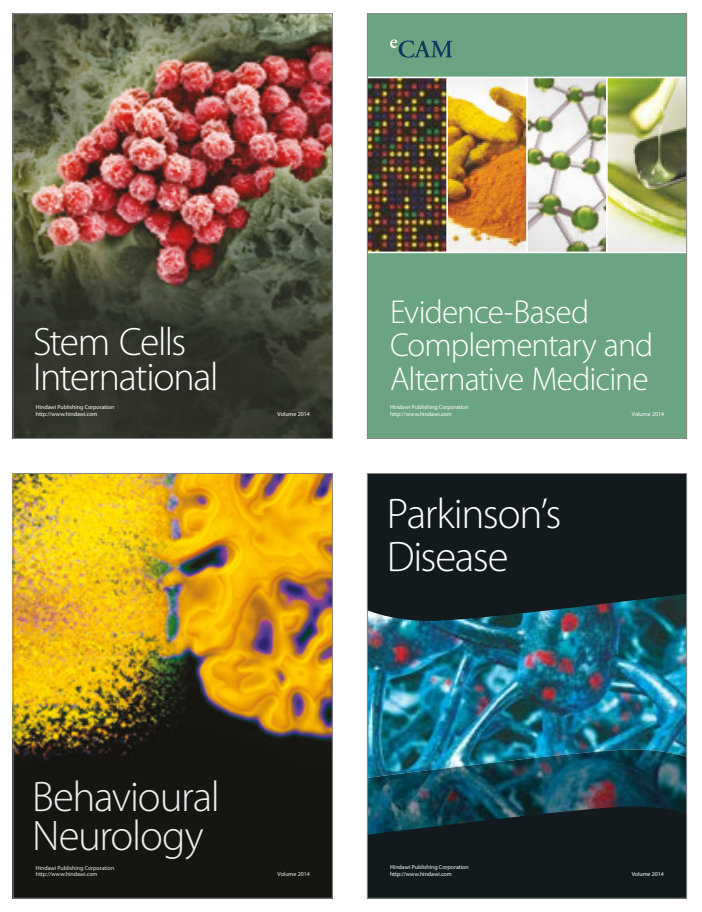
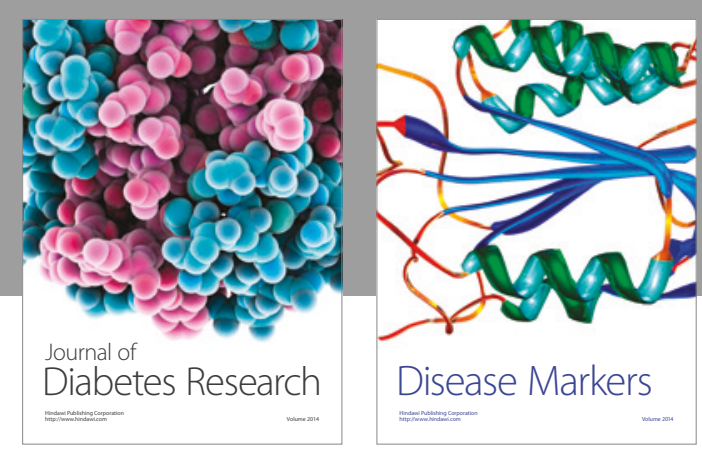

Disease Markers
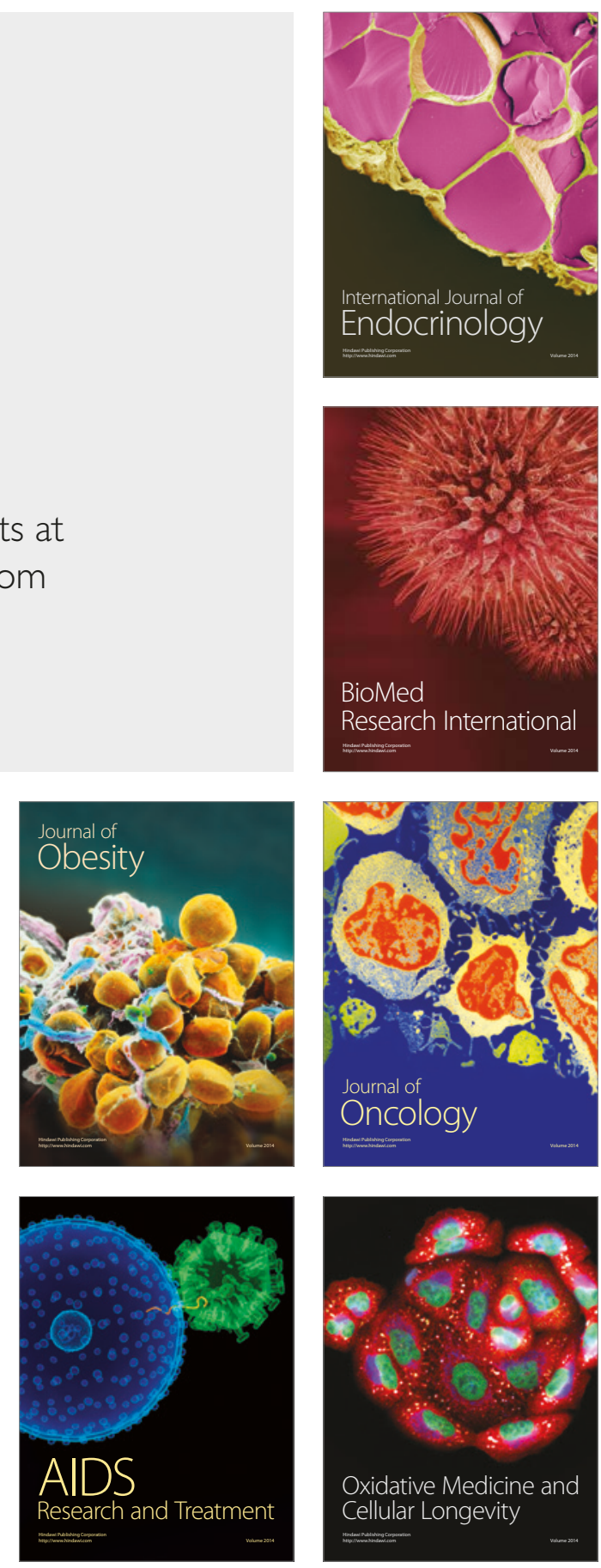